\title{
Assistência em reabilitação para vítimas de acidentes e violência: a situação dos municípios em Pernambuco, Brasil
}

\author{
Rehabilitation care for victims of accidents and violence: \\ the situation of the municipal districts in Pernambuco, Brazil
}

${ }^{1}$ Departamento de Estudos em Saúde Coletiva, Centro de Pesquisas Aggeu Magalhães, Fundação Oswaldo Cruz (Fiocruz). Av. Moraes Rego s/n, Campus da UFPE. 50670-420 Recife PE. mluizatimoteo@bol.com.br ${ }^{2}$ Departamento de Ensino, Instituto Fernandes Figueira, Fundação Oswaldo Cruz (Fiocruz)

${ }^{3}$ Centro Latino Americano de Estudos de Violência e Saúde Jorge Carelli, Claves (Fiocruz)

\begin{abstract}
A diagnostic analysis of care was conducted for rehabilitation of victims of accidents and violence in municipal districts of Pernambuco, Brazil, with over 100 thousand inhabitants. A cross-sectional and descriptive study was carried out. The principles of the triangulation method were followed because the study required the input of various informers and used a variety of collection techniques. An interview was conducted with the municipal manager of each district to map out and select the services to be analyzed. Questionnaires were applied to the managers of the services for identification of the unit, characterization of the activities and description of the structure. The results revealed that more than half of the services involved group care, prevention of relapses and neuropsychomotor stimulation. The team numbered 273 health professionals, with physiotherapists being the largest group (34.8\%), followed by psychologists (13.5\%), and speech therapists (12.8\%). Only one municipal district supplied ortheses, prostheses and auxiliary equipment for locomotion. Deficits of some types of services and discrepancies in the distribution of such services were observed in the different areas of the state.
\end{abstract}

Key words Violence, Accidents, Health services, Rehabilitation, Health policy
Resumo Foi realizada uma análise diagnóstica da assistência em reabilitação para vítimas de acidentes e de violência em municípios de Pernambuco, Brasil, com mais de 100 mil habitantes. O desenho da investigação foi transversal e descritivo. Por contemplar a visão de vários informantes e empregar uma variedade de técnicas de coleta, foram adotados os princípios da triangulação de métodos. Foram investigados sete municípios e 21 Serviços de Reabilitação, com a aplicação de entrevista ao gestor municipal, para mapear e selecionar os serviços a serem analisados, e de questionário aos gerentes dos serviços, para identificação da unidade, caracterização das atividades e descrição da estrutura. Os resultados apontaram que mais da metade dos serviços realizam apenas atendimentos em grupo, prevenção de sequelas e estimulação neuropsicomotora. O quadro profissional dos 21 Serviços é composto por 273 pessoas, o Fisioterapeuta tem maior representatividade (34,8\%), seguido do Psicólogo (13,5\%) e do Fonoaudiólogo (12,8\%). Apenas um município realiza dispensação de órteses, próteses e meios auxiliares para locomoção. Foram observados déficits de oferta de alguns tipos de atendimento e discrepâncias na distribuição geográfica desses serviços, nas diferentes regiões do Estado.

Palavras-chave Violência, Acidentes, Serviços de saúde, Reabilitação, Política de saúde 


\section{Introdução}

Nos últimos anos, a constituição de políticas públicas vem configurando diretrizes que apontam para a necessidade de organização dos serviços de saúde em consonância com o perfil epidemiológico da população brasileira, no caso em questão: a organização dos serviços de reabilitação para vítimas de acidentes e violência.

Os acidentes e as violências ocupam a terceira causa de morte no Brasil, sendo a primeira nas faixas etárias de 5 a 49 anos. No que se refere aos homicídios verificam-se importantes diferenças entre as regiões brasileiras, a Região Nordeste apresentou as maiores taxas de homicídios por arma de fogo. Entre as capitais, sete apresentaram taxas maiores que 90/100 mil habitantes, entre elas Recife, considerada uma das capitais mais violentas do Brasil ${ }^{1-4}$.

No aspecto morbidade, em 2006, o Brasil registrou 822.412 internações por acidentes e violência, estando os acidentes de transporte (15\%) e as agressões $(5,9 \%)$ entre as principais causas de lesão $\mathrm{O}^{1,3}$. Para cada morto em acidente de transporte, cerca de 13 ficam feridos, requerendo, em maior ou menor grau, assistência hospitalar e de reabilitação ${ }^{5}$.

Estudo realizado em 171 pacientes atendidos no ambulatório de Lesão Medular do Lar Escola São Francisco - EPM/UFSP, entre 1999 e 2001, identificou que $30,1 \%$ das lesões tinham sido causadas por ferimento por arma de fogo e 17,5\% em decorrência de quedas. Além disso, o tempo médio de internação foi de 54,5 dias e o tempo médio entre a alta hospitalar e a reabilitação foi de 22,4 meses $^{6}$.

Para o sistema de saúde, as consequências da violência e acidentes, dentre outros aspectos, se traduzem em aumento de gastos com emergências, assistência clínica e reabilitação, sendo que esta última envolve procedimentos muito mais custosos. Estima-se que cerca de 3,3\% do PIB brasileiro é gasto com os custos diretos da violência, elevando-se para $10,5 \%$ quando se incluem os custos indiretos e as transferências de recursos ${ }^{7}$.

$\mathrm{Na}$ tentativa de enfrentamento da questão, o Ministério da Saúde promulgou a Portaria MS/ GM no737, de 16/05/01, instituindo a Política Nacional de Redução da Morbimortalidade por Acidentes e Violência (PNRMAV). Com isso, o tema passou a ser respaldado por uma política específica ${ }^{2}$.

A PNRMAV orienta, por meio das suas diretrizes, a estruturação e a consolidação dos três níveis de atenção, desde o pré-hospitalar fixo e móvel, o hospitalar e a reabilitação. A assistência em reabilitação deve incluir não só a recuperação do indivíduo, mas a inclusão social. Assim, uma das diretrizes prevê a assistência em reabilitação, no intuito de favorecer o alcance da autonomia da vítima dentro da nova situação de saúde ${ }^{8}$.

É necessário considerar que o campo assistencial voltado para a reabilitação de pessoas com deficiência, temporária ou permanente, abrange diferentes modelos. Muitos serviços objetivam apenas o abrigo e o suprimento de necessidades básicas, com limitações no cuidado específico em reabilitação?.

Decorridas mais de duas décadas desde as primeiras discussões, quando se buscou tratar a reabilitação como um dos componentes da atenção integral à saúde, nos anos 70, ainda se verifica a predominância das concepções e ações de reabilitação enquanto serviço especializado, incorporável exclusivamente ao setor terciário da assistência9.

Pesquisas sobre serviços de reabilitação não são sistemáticas no Brasil. Estudo da Organização Panamericana de Saúde (Opas) ${ }^{10}$, que congregou dados coletados pelos ministérios da saúde de países da América Latina e Caribe, sobre políticas, programas e serviços oferecidos, mostrou que, em 1995, não se dispunha das informações necessárias para conhecer a magnitude do problema das incapacidades nessa área geográfica ${ }^{11}$.

Dados da Opas, da Organização Mundial de Saúde (OMS) e do Fundo das Nações Unidas para a Infância (Unicef) constituem as principais informações que alimentam os debates no campo do planejamento e organização de serviços de reabilitação nos países menos desenvolvidos. Muito do que se discutiu, nos últimos anos, em relação à reabilitação no Brasil, teve por base poucas informações sobre a realidade local, captadas de forma assistemática, e avaliações, considerações e recomendações dos organismos citados ${ }^{12}$.

Já existem indicativos acerca do quanto ainda precisa avançar a organização dos serviços de reabilitação no Brasil. Estudo desenvolvido por Minayo e Deslandes ${ }^{13}$, em cinco capitais brasileiras, a partir da análise diagnóstica da PNRMAV, apontou que os serviços de reabilitação, comparados aos de atenção pré-hospitalar e hospitalar, são, sem dúvida, os que mais evidenciam déficit de oferta, entre outros aspectos, em organização e estrutura em todas as capitais estudadas, sendo, portanto, a área de maior fragilidade no processo de implantação da Política Nacional.

Alguns estudos avaliando a assistência em reabilitação foram realizados sobre a Política de Saú- 
de Auditiva em Pernambuco ${ }^{14}$, a atenção em Saúde Mental em São Paulo ${ }^{15}$, e um terceiro enfocando a distribuição dos serviços de reabilitação no Rio de Janeiro ${ }^{16}$. Porém, são inexistentes os estudos sobre assistência em reabilitação física.

Diante da ausência destas informações, o que se configura como empecilho à prevenção das incapacidades, ao planejamento das ações e à garantia e cumprimento do princípio fundamental denominado "saúde integral", buscar-se-á conhecer, em maior profundidade, a organização e a estrutura da assistência em reabilitação física nos municípios de mais de 100 mil habitantes, em Pernambuco, à luz da PNRMAV, no propósito de contribuir para um maior conhecimento do tema.

\section{Métodos}

O presente estudo é um desdobramento e aplicação da pesquisa e metodologia desenvolvida pelo Centro Latino-Americano de Estudo em Violência e Saúde Jorge Carelli (Claves/ENSP/Fiocruz) na pesquisa Análise Diagnóstica da Política Nacional de Saúde para Redução de Acidentes e Violências, realizada em cinco capitais brasileiras.

O desenho da investigação atende as características de um estudo transversal, descritivo. Do ponto de vista conceitual, a investigação seguiu os princípios da triangulação de métodos, que visa: (a) a combinação e o cruzamento de múltiplos pontos de vista; (b) a tarefa conjunta de pesquisadores com formação diferenciada; (c) a visão de vários informantes e (d) o emprego de uma variedade de técnicas de coleta de dados que acompanha o trabalho de investigação ${ }^{13}$.

A investigação foi desenvolvida no Estado de Pernambuco, tendo sido selecionados os municípios com população superior a 100 mil habitantes. A opção de trabalhar com cidades desse porte se deu por estas apresentarem as mais altas taxas de violência de Pernambuco, tomando como referência o diagnóstico realizado com os dados do Sistema de Informações Policiais (Infopol) da Secretaria de Defesa Social, do Sistema de Informação da Mortalidade (SIM) e do Sistema de Informação Hospitalar (SIH), do Departamento de Informática do SUS (Datasus), apresentados no diagnóstico do Plano Estadual de Segurança Pública, denominado "Pacto pela Vida", do Governo de $\mathrm{PE}^{17}$.

Barata e Ribeiro ${ }^{18}$ relataram a importância das aglomerações urbanas como fator predisponente ou facilitador para a ocorrência de homicídios, a partir de análises de porte populacional.
Após a seleção dos municípios, procedeu-se a identificação das unidades de saúde. Foi solicitado às secretarias de saúde de cada cidade que enviassem a relação dos serviços de reabilitação, da rede própria e conveniada, que realizam atendimento, reconhecido pelos gestores como regular e rotineira, para vítimas de acidentes e violências (Quadro 1).

Para a análise diagnóstica dos serviços de reabilitação, tomou-se por base as orientações da quinta diretriz da PNRMAV e a Portaria no 818/ 2001, que cria mecanismos para a organização e a implantação de Redes Estaduais de Assistência à Pessoa Portadora de Deficiência Física ${ }^{13}$.

A análise diagnóstica será entendida como preconizam Minayo e Deslandes" ${ }^{13}$ "Uma forma de olhar compreensiva e interpretativa que permite dar conta dos êxitos e das dificuldades dos sistemas de saúde para atender à crescente demanda e às mudanças qualitativas das lesões e traumas provenientes das violências e acidentes".

A investigação desenvolveu-se a partir dos seguintes procedimentos: entrevista semiestruturada com o gestor municipal responsável pela assistência em reabilitação para mapear e selecionar os serviços a serem analisados; aplicação de questionário aos gerentes dos serviços/programas identificados na fase anterior, contemplando a identificação da unidade, a caracterização das atividades desenvolvidas, a descrição da estrutura existente e a organização dos serviços.

A análise quantitativa usou a estatística descritiva (estimativa de frequências e percentuais). As entrevistas foram transcritas e interpretadas, segundo os princípios da análise de conteúdo, a partir de sua modalidade temática. A análise de conteúdo priorizou a interpretação hermenêutica dos sentidos latentes e manifestos nos relatos, em detrimento de codificações estatísticas de sua ocorrência ${ }^{19}$. Os entrevistados foram identificados por letras e números sequenciais (p. ex., E1, E2...).

A pesquisa foi aprovada pelo Comitê de Ética do Centro de Pesquisas Aggeu Magalhães, e respeitadas as recomendações da resolução do CNS n¹96/96.

\section{Resultados}

O estudo pretendeu investigar os 10 municípios pernambucanos que em 2007 tinham uma população superior a 100 mil habitantes (Quadro 1). Contudo, foram excluídos três deles por não referirem nenhum serviço de reabilitação voltado para a assistência a vítimas de acidentes e vio- 
Quadro 1. Municípios do Estado de Pernambuco com mais de 100 mil habitantes e serviços de reabilitação que assistem vítimas de acidentes e violência em 2007

\begin{tabular}{|c|c|c|}
\hline Município & $\begin{array}{l}\text { População/ } \\
\text { hab em } 2007\end{array}$ & Serviços de reabilitação indicados \\
\hline \multicolumn{3}{|c|}{ Ia Geres Mesorregião Metropolitana } \\
\hline Recife & 1.528 .970 & $\begin{array}{l}\text { - Centro de Reabilitação do DSI } \\
\text { - Centro de Saúde Mário Ramos } \\
\text { - Centro Social Urbano Bido Krause } \\
\text { - Policlínica Agamenon Magalhães } \\
\text { - Policlínica Amury Coutinho } \\
\text { - Associação de Apoio a Criança Deficiente (AACD) }\end{array}$ \\
\hline Jaboatão dos Guararapes & 661.901 & $\begin{array}{l}\text { - Centro de Reabilitação e Fisioterapia } \\
\text { PROSAD/CASA } \\
\text { - Centro de Reabilitação e Fisioterapia } \\
\text { Francisco Loureiro } \\
\text { - Centro de Reabilitação e Fisioterapia } \\
\text { Antônio Caldas de Sá Barreto } \\
\text { - Centro de Reabilitação e Fisioterapia Distrito I } \\
\text { - Centro de Reabilitação e Fisioterapia Distrito } \\
\text { V Regional } \\
\text { - CENAME Saúde }\end{array}$ \\
\hline Olinda & 390.455 & Centro de Reabilitação de Olinda \\
\hline Paulista & 405.308 & Sem indicação \\
\hline Cabo de Santo Agostinho & 175.049 & Sem indicação \\
\hline Camaragibe & 153.627 & Núcleo de Reabilitação \\
\hline \multicolumn{3}{|l|}{ Ia Geres Mesorregião Mata } \\
\hline Vitória de Santo Antão & 126.764 & $\begin{array}{l}\text { - Reabilitação Hospital João Murilo } \\
\text { - Reabilitação Hospital Santa Maria }\end{array}$ \\
\hline \multicolumn{3}{|c|}{ IVa Geres Mesorregião Agreste } \\
\hline Caruaru & 287.611 & $\begin{array}{l}\text { - Casa Henrique } \\
\text { - Policlínica da Terceira Idade e Fisioterapia } \\
\text { - Reabilitação do Hospital Regional do Agreste }\end{array}$ \\
\hline \multicolumn{3}{|l|}{ Va Geres Mesorregião Agreste } \\
\hline Garanhuns & 130.008 & $\begin{array}{l}\text { - Reabilitação (HDRM) } \\
\text { - Centro de Reabilitação de Garanhuns }\end{array}$ \\
\hline \multicolumn{3}{|c|}{ VIII' ${ }^{a}$ Geres Mesorregião São Francisco } \\
\hline Petrolina & 266.269 & Sem indicação \\
\hline
\end{tabular}

lência, a saber: Paulista, Petrolina e Cabo de Santo Agostinho. Assim, a pesquisa investigou sete municípios e 21 serviços de reabilitação.
Dos 21 serviços analisados, tomando como base a classificação da Portaria no 818/01, que define os níveis de assistência segundo a sua com- 
plexidade, 15 foram classificados como de primeiro nível, 03 como de nível intermediário e 03 como de referência.

Com relação ao tipo de atendimento prestado, destaca-se que os atendimentos em grupo são praticados por pouco mais da metade das unidades de saúde, e em duas cidades esse atendimento não é realizado. A prevenção de sequelas é uma atividade oferecida por todos os serviços, exceto em Jaboatão dos Guararapes, e a estimulação neuropsicomotora é realizada por quase $70 \%$ dos serviços, exceto em Jaboatão dos Guararapes e Vitória de Santo Antão. Em quatro municípios, Recife, Caruaru, Vitória de Santo Antão, e Garanhuns, alguns serviços não realizam ações de orientação à família, e apenas Recife possui serviços que realizam avaliação funcional e diagnóstico de eletromiografia e potenciais evocados (Tabela 1).

Algumas especificidades nos atendimentos em reabilitação estão explicitadas na Tabela 2. Os atendimentos domiciliares e o acompanhamento pósalta são realizados apenas nos municípios de Re- cife e Jaboatão dos Guararapes. Constituem práticas inexistentes nos demais municípios. A preparação para a alta, o suporte para o convívio familiar e as informações sobre os direitos das pessoas deficientes são práticas realizadas por quase todos os municípios, porém em poucos serviços (Tabela 2). Os serviços foram questionados sobre a adequação e a suficiência das ações, o que acarretou num descompasso: quando existia o tipo de atendimento, o mesmo não atendia na adequação e na suficiência desejáveis.

Dentre os serviços, mais de $80 \%$ realizam ações de avaliação, prescrição, treino e acompanhamento para órteses, próteses e meios auxiliares de locomoção, exceto Jaboatão dos Guararapes e Vitória de Santo Antão (Tabela 1). Porém, o único município que faz dispensação de órteses, próteses e meios auxiliares para locomoção é o de Recife, em dois serviços (Tabela 3).

Nos sete municípios estudados, 273 profissionais trabalham em serviços de reabilitação. As categorias profissionais com maior representatividade nos serviços foram as de Fisioterapeu-

Tabela 1. Distribuição dos serviços de reabilitação, segundo ações realizadas em municípios com mais de 100 mil habitantes Pernambuco, 2008.

\begin{tabular}{|c|c|c|c|c|c|c|c|c|c|c|c|c|c|c|}
\hline \multirow{2}{*}{ Ações } & \multicolumn{2}{|c|}{$\begin{array}{l}\text { Recife } \\
\mathrm{N}=6\end{array}$} & \multicolumn{2}{|c|}{$\begin{array}{c}\text { Olinda } \\
\mathrm{N}=1\end{array}$} & \multicolumn{2}{|c|}{$\begin{array}{c}\text { Camaragibe } \\
\mathrm{N}=1 \\
\end{array}$} & \multicolumn{2}{|c|}{$\begin{array}{c}\text { Caruaru } \\
\mathrm{N}=3\end{array}$} & \multicolumn{2}{|c|}{$\begin{array}{l}\text { Vitória } \\
\mathrm{N}=2\end{array}$} & \multicolumn{2}{|c|}{$\begin{array}{c}\text { Jaboatão } \\
\quad \mathrm{N}=5\end{array}$} & \multicolumn{2}{|c|}{$\begin{array}{c}\text { Garanhuns } \\
\mathrm{N}=2 \\
\end{array}$} \\
\hline & $\mathrm{N}$ & $\%$ & $\mathrm{~N}$ & $\%$ & $\mathrm{~N}$ & $\%$ & $\mathrm{~N}$ & $\%$ & $\mathrm{~N}$ & $\%$ & $\mathbf{N}$ & $\%$ & $\mathrm{~N}$ & $\%$ \\
\hline Atendimento em grupo & 5 & 83,3 & 1 & 100,0 & 1 & 100,0 & 0 & - & 0 & - & 5 & 100,0 & 1 & 50,0 \\
\hline $\begin{array}{l}\text { Prevenção de sequelas e } \\
\text { incapacidades secundárias }\end{array}$ & 6 & 100,0 & 1 & 100,0 & 1 & 100,0 & 3 & 100 & 2 & 100,0 & 0 & - & 2 & 100,0 \\
\hline Estimulação neuropsicomotora & 5 & 83,3 & 1 & 100,0 & 1 & 100,0 & 2 & 66,7 & 0 & - & 2 & 40,0 & 2 & 100,0 \\
\hline Orientação familiar & 3 & 50,0 & 1 & 100,0 & 1 & 100,0 & 2 & 66,7 & 1 & 50,0 & 5 & 100,0 & 1 & 50,0 \\
\hline Aval. Médica, clinica e functional & 5 & 50,0 & 1 & 100,0 & 0 & - & 2 & 66,7 & 2 & 100,0 & 2 & 40,0 & 1 & 50,0 \\
\hline Aval. Clinica especializada & 5 & 50,0 & 1 & 100,0 & 1 & 100,0 & 3 & 100,0 & 2 & 100,0 & 3 & 60,0 & 2 & 100,0 \\
\hline Aval. e atend. em fisioterapia & 6 & 100,0 & 1 & 100,0 & 1 & 100,0 & 3 & 100,0 & 2 & 100,0 & 4 & 80,0 & 2 & 100,0 \\
\hline $\begin{array}{l}\text { Aval. e atend. em terapia } \\
\text { ocupacional }\end{array}$ & 4 & 66,7 & 0 & - & 1 & 100,0 & 0 & - & 0 & - & 3 & 60,0 & 0 & - \\
\hline Aval. e atend. em fonoaudiologia & 4 & 66,7 & 1 & 100,0 & 1 & 100,0 & 2 & 66,7 & 0 & - & 5 & 100,0 & 2 & 100,0 \\
\hline Aval. e atend. em psicologia & 4 & 66,7 & 1 & 100,0 & 1 & 100,0 & 1 & 33,3 & 1 & 50,0 & 4 & 80,0 & 1 & 50,0 \\
\hline Aval. e atend. em serviço social & 4 & 66,7 & 0 & - & 1 & 100,0 & 1 & 33,3 & 1 & 50,0 & 3 & 60,0 & 1 & 50,0 \\
\hline Aval. e atend. em enfermagem & 3 & 50,0 & 0 & - & 0 & - & 2 & 66,7 & 2 & 100,0 & 1 & 20,0 & 0 & - \\
\hline Aval. e atend. em nutrição & 3 & 50,0 & 0 & - & 0 & - & 2 & 66,7 & 1 & 50,0 & 0 & - & 0 & - \\
\hline $\begin{array}{l}\text { Atend. medicamentoso com } \\
\text { dispensação de remedios }\end{array}$ & 1 & 16,7 & 0 & - & 1 & 100,0 & 1 & 33,3 & 1 & 50,0 & 0 & - & 0 & - \\
\hline $\begin{array}{l}\text { Aval. funcional e de diagnóstico de } \\
\text { eletroneuromiografiae potenciais } \\
\text { evocados }\end{array}$ & 2 & 33,3 & 0 & - & 0 & - & 0 & - & 0 & - & 0 & - & 0 & - \\
\hline Aval. Urodinâmica & 0 & - & 0 & - & 0 & - & 0 & - & 0 & - & 0 & - & 0 & - \\
\hline $\begin{array}{l}\text { Atend. Clínico nas diversas } \\
\text { especialidades médicas }\end{array}$ & 3 & 50,0 & 0 & - & 0 & - & 2 & 66,7 & 1 & 50,0 & 1 & 20,0 & 1 & 50,0 \\
\hline Atend. Cirúrgico & 0 & - & 0 & - & 0 & - & 1 & 33,3 & 2 & 100,0 & 0 & - & 0 & - \\
\hline
\end{tabular}




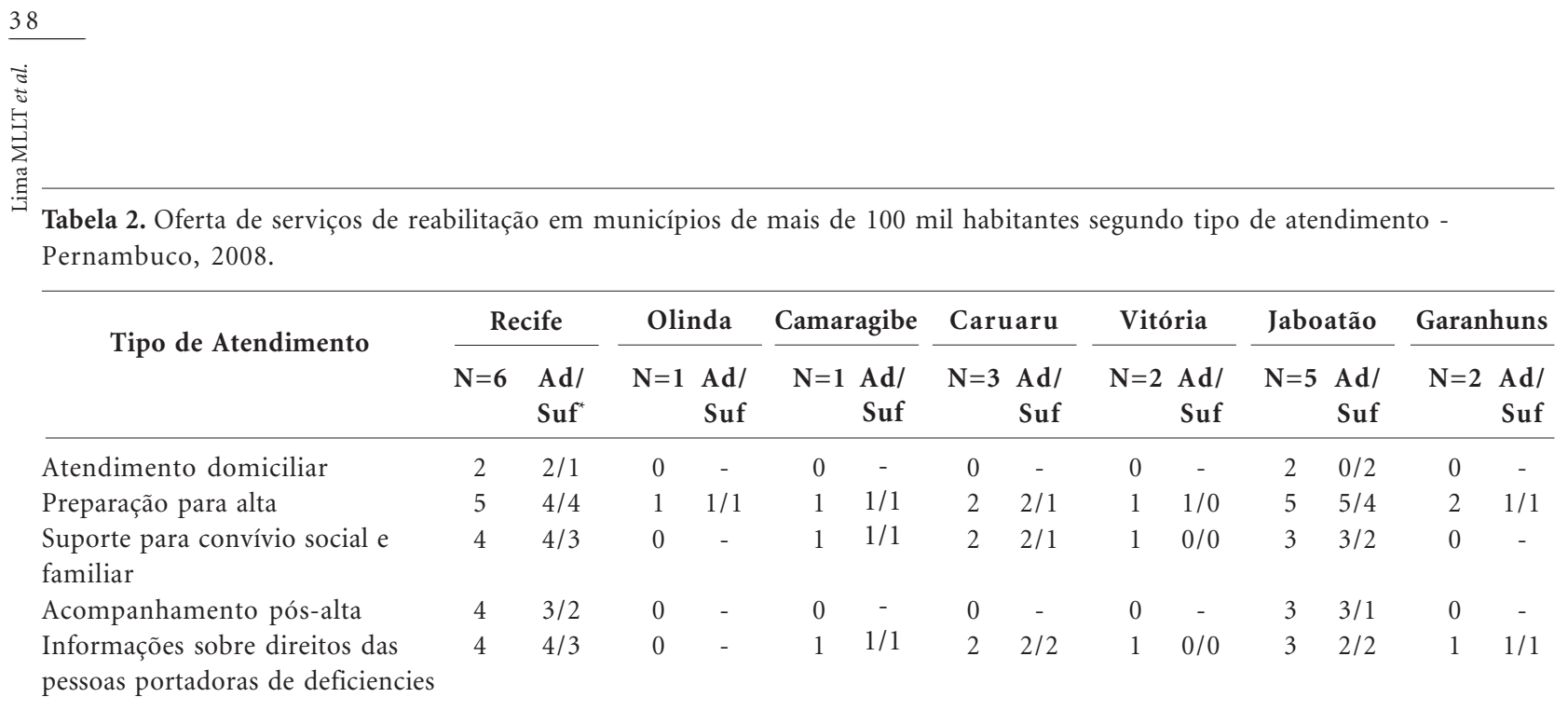

* Número de serviços que conideram o atendimento Adequado/Suficiente

Tabela 3. Distribuição dos serviços de reabilitação em municípios de mais de 100 mil habitantes segundo a provisão de recursos tais como órteses, próteses e meios auxiliares de locomoção - Pernambuco, 2008.

\begin{tabular}{|c|c|c|c|c|c|c|c|c|c|c|c|c|c|c|}
\hline \multirow{2}{*}{ Tipo de Atendimento } & \multicolumn{2}{|c|}{$\begin{array}{l}\text { Recife } \\
N=6\end{array}$} & \multicolumn{2}{|c|}{$\begin{array}{c}\text { Olinda } \\
\mathrm{N}=1\end{array}$} & \multicolumn{2}{|c|}{$\begin{array}{c}\text { Camaragibe } \\
\qquad \mathrm{N}=1\end{array}$} & \multicolumn{2}{|c|}{$\begin{array}{c}\text { Caruaru } \\
\mathrm{N}=3\end{array}$} & \multicolumn{2}{|c|}{$\begin{array}{l}\text { Vitória } \\
\mathrm{N}=2\end{array}$} & \multicolumn{2}{|c|}{$\begin{array}{c}\text { Jaboatão } \\
\qquad \mathrm{N}=\mathbf{5}\end{array}$} & \multicolumn{2}{|c|}{$\begin{array}{c}\text { Garanhuns } \\
\mathrm{N}=2 \\
\end{array}$} \\
\hline & $\mathbf{N}$ & $\%$ & $\mathbf{N}$ & $\%$ & $\mathbf{N}$ & $\%$ & $\mathbf{N}$ & $\%$ & $\mathbf{N}$ & $\%$ & $\mathrm{~N}$ & $\%$ & $\mathbf{N}$ & $\%$ \\
\hline \multicolumn{15}{|l|}{ Órtese } \\
\hline Avalia indicação & 4 & 66,6 & 1 & 100,0 & 1 & 100,0 & \multirow{5}{*}{$\begin{array}{l}2 \\
3 \\
3 \\
0\end{array}$} & \multirow{5}{*}{$\begin{array}{c}100,0 \\
66,7 \\
100,0 \\
100,0 \\
-\end{array}$} & 1 & 50,0 & 2 & 40,0 & 2 & 100,0 \\
\hline Presecreve utilização & 5 & 83,3 & 1 & 100,0 & 1 & 100,0 & & & 1 & 50,0 & 2 & 40,0 & 2 & 100,0 \\
\hline Treina para o uso & 4 & 66,6 & 1 & 100,0 & 1 & 100,0 & & & 1 & 50,0 & 4 & 80,0 & 2 & 100,0 \\
\hline Faz acompanhamneto & 5 & 83,3 & 1 & 100,0 & 0 & 100,0 & & & 1 & 50,0 & 3 & 60,0 & 2 & 100,0 \\
\hline Faz dispensação & 2 & 33,3 & 0 & - & 0 & - & & & 0 & - & 0 & - & 0 & - \\
\hline \multicolumn{15}{|l|}{ Próteses } \\
\hline Avalia indicação & 3 & 50,0 & 1 & 100,0 & 1 & 100,0 & \multirow{5}{*}{$\begin{array}{l}2 \\
3 \\
3 \\
0\end{array}$} & \multirow{5}{*}{$\begin{array}{c}100,0 \\
33,3 \\
100,0 \\
100,0 \\
-\end{array}$} & 1 & 50,0 & 2 & 40,0 & 2 & 100,0 \\
\hline Presecreve utilização & 4 & 66,6 & 1 & 100,0 & 1 & 100,0 & & & 0 & - & 2 & 40,0 & 2 & 100,0 \\
\hline Treina para o uso & 4 & 66,6 & 0 & - & 1 & 100,0 & & & 2 & 100,0 & 3 & 60,0 & 2 & 100,0 \\
\hline Faz acompanhamneto & 5 & 83,3 & 1 & 100,0 & 0 & - & & & 1 & 50,0 & 3 & 60,0 & 2 & 100,0 \\
\hline Faz dispensação & 2 & 33,3 & 0 & - & 0 & - & & & 0 & - & 0 & - & 0 & - \\
\hline \multicolumn{15}{|l|}{ Meios auxiliares de locomoção } \\
\hline Avalia indicação & 4 & 66,6 & 1 & 100,0 & 1 & 100,0 & \multirow{5}{*}{$\begin{array}{l}3 \\
0\end{array}$} & \multirow{5}{*}{$\begin{array}{c}33,3 \\
100,0 \\
100,0 \\
-\end{array}$} & 2 & 100,0 & 2 & 40,0 & 1 & 50,0 \\
\hline Presecreve utilização & 5 & 83,3 & 1 & 100,0 & 1 & 100,0 & & & 1 & 50,0 & 2 & 40,0 & 1 & 50,0 \\
\hline Treina para o uso & 4 & 66,6 & 1 & 100,0 & 1 & 100,0 & & & 2 & 100,0 & 4 & 80,0 & 1 & 50,0 \\
\hline Faz acompanhamneto & 5 & 83,3 & 1 & 100,0 & 0 & - & & & 2 & 100,0 & 3 & 60,0 & 1 & 50,0 \\
\hline Faz dispensação & 3 & 50,0 & 0 & - & 0 & - & & & 0 & - & 0 & - & 0 & - \\
\hline
\end{tabular}

tas $(34,8 \%)$, Psicólogos $(13,5 \%)$ e Fonoaudiólogos $(12,8 \%)$. O Fisiatra é um profissional inexistente nos serviços do Estado de Pernambuco, e os Farmacêuticos e os Nutricionistas têm pequeníssima participação nas equipes. Destaca-se ainda a inexistência de Assistente Social nos serviços de Olinda e de Caruaru (Tabela 4).

\section{Discussão}

Em Pernambuco, a distribuição dos municípios estudados mostra a concentração dos serviços de reabilitação na Mesorregião Metropolitana do Recife (14), seguida da Mesorregião Agreste (5) e da Mata (2). As regiões mais distantes da capital, e com grandes áreas territoriais, não dispõem de serviços de reabilitação para assistência às vítimas de acidentes e violência. A única cidade in- 
Tabela 4. Distribuição dos profissionais nos serviços/programas de reabilitação nos municípios de mais de 100 mil habitantes - Pernambuco, 2008.

\begin{tabular}{|c|c|c|c|c|c|c|c|c|c|}
\hline \multirow[b]{2}{*}{ Profissionais } & \multirow{2}{*}{$\frac{\text { Recife }}{\mathrm{N}}$} & \multirow{2}{*}{$\frac{\text { Olinda }}{\mathrm{N}}$} & \multirow{2}{*}{$\frac{\text { Camaragibe }}{\mathrm{N}}$} & \multirow{2}{*}{$\frac{\text { Caruaru }}{\mathrm{N}}$} & \multirow{2}{*}{$\frac{\text { Vitória }}{\mathrm{N}}$} & \multirow{2}{*}{$\frac{\text { Jaboatão }}{\mathrm{N}}$} & \multirow{2}{*}{$\frac{\text { Garanhuns }}{\mathrm{N}}$} & \multicolumn{2}{|c|}{ Total } \\
\hline & & & & & & & & $\mathbf{N}$ & $\%$ \\
\hline Médico fisiatra & 0 & 0 & 0 & 0 & 0 & 0 & 0 & 0 & - \\
\hline Fisioterapeuta & 38 & 8 & 5 & 13 & 5 & 17 & 9 & 95 & 34,8 \\
\hline Fonoaudiólogo & 15 & 3 & 2 & 3 & 0 & 10 & 2 & 35 & 12,8 \\
\hline Enfermeiro & 4 & 0 & 0 & 2 & 1 & 0 & 1 & 8 & 2,9 \\
\hline Assistente social & 7 & 0 & 1 & 0 & 2 & 5 & 1 & 16 & 5,9 \\
\hline Psicólogo & 12 & 1 & 2 & 1 & 2 & 16 & 3 & 37 & 13,5 \\
\hline Terapeuta ocupacional & 16 & 0 & 2 & 0 & 0 & 5 & 0 & 23 & 8,4 \\
\hline Nutricionista & 3 & 0 & 0 & & 2 & 0 & 1 & 6 & 2,2 \\
\hline Farmacêutico & 3 & 0 & 0 & 0 & 0 & 0 & 1 & 4 & 1,4 \\
\hline Responsável técnico & 4 & 1 & 1 & 3 & 2 & 8 & 1 & 20 & 7,3 \\
\hline Técnico/auxiliar de enfermagem & 4 & 2 & 1 & 6 & 3 & 4 & 9 & 29 & 10,6 \\
\hline Total & 106 & 15 & 14 & 28 & 17 & 65 & 28 & 273 & 100,0 \\
\hline
\end{tabular}

cluída, pelo seu porte populacional, para representar a Mesorregião do São Francisco, não indicou a existência de serviços voltados para essa assistência, e a Mesorregião do Sertão não possui nenhum município com mais de 100 mil habitantes.

É possível inferir que as populações das mesorregiões do São Francisco e Sertão estão desassistidas. É pouco provável que as pessoas que precisam de assistência em reabilitação se desloquem para a capital do Estado ou para a Região Metropolitana do Recife, uma vez que a assistência em reabilitação tem características diferentes das demais áreas e requer, quase sempre, um cuidado intenso e continuado por longo período de tempo.

Ademais, do total de 21 serviços voltados ao atendimento de vítimas de acidentes e violência, aproximadamente $66,6 \%$ se encontram na Mesorregião Metropolitana do Recife. Os três municípios com mais de 100 mil habitantes que foram inicialmente selecionados e que não dispunham de serviços de reabilitação abrigam uma população de 846.626 habitantes. Essa distribuição desigual, acrescida às possíveis dificuldades de locomoção, visuais, auditivas e de comunicação dos potenciais usuários, limitará ainda mais o uso desses serviços.

Pesquisa desenvolvida, em 2005, em cinco capitais brasileiras, objetivando uma análise diagnóstica dos serviços que assistem vítimas de acidentes e violências, revelou que há insuficiência de serviços de reabilitação, em todas as capitais estudadas ${ }^{20}$. No Estado do Rio de Janeiro, 73\% do atendimento em reabilitação voltado à pes- soa portadora de deficiência estão concentrados na Região Metropolitana I, mostrando a desigualdade da oferta e acesso da população a um serviço indispensável para a consecução da integralidade na saúde ${ }^{16}$.

Em consonância com essas informações está o relato da Coordenação da Área Técnica de Saúde da Pessoa com Deficiência do Ministério da Saúde ${ }^{21}$, que chama a atenção para a atual situação brasileira quanto à reabilitação, ao afirmar que "a maioria dos municípios brasileiros não tem adequado atendimento para reabilitação".

No que se refere a Pernambuco, tais informações são reforçadas na fala do gestor: "A estrutura de atendimento dos serviços de reabilitação para atendimento a vítimas de acidentes e violências tem sido percebida como insuficiente para a demanda que o município vem apresentando, pois está em fase de organização no que diz respeito à atenção em reabilitação". (E1)

Entre as ações desenvolvidas pelos serviços de reabilitação incluem-se os atendimentos em grupo, que não são realizados em, pelo menos, dois munícipios. O fato chama a atenção, uma vez que o atendimento em grupo é um importante recurso terapêutico para otimização dos espaços e equipamentos, para trocas e construção coletiva. E ainda mais: como os atendimentos em reabilitação podem demandar longos períodos de tempo, os atendimentos em grupo podem constituir uma estratégia que possibilita a ampliação da cobertura.

Da mesma forma que são poucos os atendimentos em grupo, são escassos os atendimentos domiciliares. Em depoimento, os gestores desta- 
cam a importância do trabalho dos Núcleos de Apoio à Saúde da Família (Nasf), no que diz respeito ao atendimento domiciliar: "O atendimento domiciliar, nós temos agora, com a implantação do Nasf, apesar do pouco tempo, são oito equipes. A gente notou que o negócio foi viabilizado". (E2)

Como mais de $50 \%$ das unidades se autoclassificaram como de nível primário, ações voltadas para o atendimento em grupo deveriam ter sido mais contempladas. A portaria $\mathrm{n}^{\circ} 818^{8}$ aponta que entre as ações realizadas pelos serviços de Reabilitação Física - Primeiro Nível de Referência Intermunicipal, e os serviços de Reabilitação Física - Nível Intermediário - devem constar atividades de atendimento em grupo (atividades educativas em saúde, grupos de orientação, modalidades terapêuticas de reabilitação e atividades da vida diária).

Também, atendimentos de preparação para a alta e suporte para o convívio familiar não são realizados por boa parte dos serviços dos municípios pernambucanos. Fato que provoca uma reflexão sobre a qualidade dessa assistência, uma vez que essas ações deveriam ter cunho obrigatório em qualquer área e nível do cuidado. A assistência à família se configura como uma medida essencial para um atendimento completo e eficaz. Essa assistência compreende ações de apoio psicossocial e orientações para a realização das atividades da vida diária ${ }^{22}$.

É importante registrar que os serviços estudados foram aqueles indicados pelas secretarias de saúde como de referência para a assistência a vítimas de acidentes e violência. No entanto, a partir do discurso de alguns gestores, é possível identificar que o próprio serviço não faz essa distinção: "Não temos conseguido quantificar, separadamente, pacientes vindos de acidentes de motos ou de outro tipo de acidente. Observamos as sequelas e não sua origem”. (E3)

Além disso, em alguns casos, a classificação em que se enquadraram, primeiro nível, nível intermediário ou referência, não condiz com o quadro funcional, as ações desenvolvidas e a estrutura existente.

Em todas as cidades estudadas há, pelo menos, um serviço que realiza a avaliação, a prescrição, o treino para o uso, o acompanhamento de órteses, próteses e meios auxiliares de locomoção. Porém, a dispensação é realizada apenas na cidade do Recife e por dois dos serviços, sendo um deles conveniado ao SUS. Esta representa uma grave lacuna na assistência em reabilitação. A portaria no $818 / 2001$ prevê que a dispensação de órteses, próteses e meios auxiliares de locomoção seja realizada por todos os serviços, seja de primeiro nível, de nível intermediário ou de referência em Medicina Física e Reabilitação.

A atual situação da dispensação de órteses, próteses e meios auxiliares de locomoção, para os municípios estudados, pode ser resumida na fala da coordenadora da Política de Reabilitação de um dos municípios: "A oferta de órteses e próteses realmente não há no município especificamente, nós encaminhamos para Recife”. (E4)

A maior representatividade da Fisioterapia e a ausência de alguns profissionais que devem obrigatoriamente compor equipes mínimas nos serviços de primeiro nível, como é o caso do Assistente Social, é bastante prejudicial e configura uma restrita possibilidade de intervenção em pacientes que requerem ações específicas e diversas diante da gravidade do seu quadro. Além disso, a inexistência, em algumas cidades, de Terapeuta Ocupacional e Fonoaudiólogo, compromete o atendimento, que deve ser multiprofissional e integral.

Estudo realizado em um hospital de referência para reabilitação de crianças, constatou que o Assistente Social e o Fisioterapeuta Motor foram os profissionais com maior demanda, $81 \%$ e $60,4 \%$ respectivamente ${ }^{23}$.

Em toda a rede estudada, é inexistente a presença de Fisiatras (médicos especializados em Medicina Física e de Reabilitação). A escassez destes profissionais pode ser confirmada pela Associação Brasileira de Medicina Física e Reabilitação que, em $2009^{24}$, registrava apenas um profissional no Estado de Pernambuco, sem a indicação do local de trabalho. Realidade aparentemente também vivida por outros estados, como Santa Catarina, onde, no início da década de 90, a Sociedade Catarinense de Fisiatria contava com apenas sete profissionais ${ }^{25}$.

Em relação aos profissionais envolvidos, é possível perceber, nas entrevistas, que pelo menos três gestores já apontam o Nasf como responsável pelo desafogamento e apoio real a algumas unidades de reabilitação. Como visto no relato: "São fisioterapeutas, farmacêuticos, assistentes sociais, educadores físicos e psicólogos. Então, a gente tem conseguido dar uma qualidade maior de atendimento e esvaziar um pouco o centro de reabilitação". (E3)

Os Nasfs, instituídos pela portaria no 154 / GM, de 2008, têm como objetivo ampliar a abrangência e o escopo das ações de atenção básica, bem como sua resolubilidade, apoiando a inserção da estratégia de Saúde da Família na rede de 
serviços e o processo de territorialização e regionalização a partir da atenção básica ${ }^{26}$.

Para a adesão e a implantação dos Nasfs pelos gestores municipais, é necessário elaborar um projeto onde, entre outras informações, constem as principais ações planejadas e os profissionais pleiteados. Os municípios submetem os projetos ao Conselho Municipal de Saúde, e a Secretaria Estadual de Saúde submete o pleito do município à apreciação da Comissão Intergestores Bipartite. Após as aprovações serão repassados os incentivos financeiros do Fundo Nacional de Saúde para o Fundo Municipal de Saúde, de acordo com a modalidade de Nasf.

Os núcleos devem ser constituídos por, no mínimo, cinco profissionais, definidos pelos gestores municipais, dentre as seguintes ocupações: Médico Acupunturista, Assistente Social, Profissional de Educação Física, Farmacêutico, Fisioterapeuta, Fonoaudiólogo, Médico Ginecologista, Médico Homeopata, Nutricionista, Médico Pediatra, Psicólogo, Médico Psiquiatra e Terapeuta Ocupacional ${ }^{26}$.

Assim, os gestores têm entendido que os Nasfs poderão reforçar o programa de reabilitação baseado na atuação da comunidade, uma vez que preconiza a reabilitação das pessoas com deficiência no âmbito das unidades básicas de saúde, com o apoio de profissionais de saúde e de familiares ${ }^{27}$.

\section{Conclusões}

A análise diagnóstica dos serviços de assistência em reabilitação física em municípios com mais de 100 mil habitantes de Pernambuco, em relação à distribuição dos serviços sob o ponto de vista geográfico, apontou maior concentração na Mesorregião metropolitana do Recife.

Da mesma forma, com relação à distribuição de profissionais, algumas áreas específicas não são contempladas nos serviços de reabilitação, indicando o descumprimento das recomendações da portaria no 818 , de 2001, e o descompro- misso com a cobertura e a integralidade deste tipo de assistência.

Se a problemática dos elevados índices de acidentes e violência corresponde a um dos principais indicadores de mortalidade e morbidade nessa região, ao que parece os Serviços não percebem esta realidade e não desenvolvem as ações que se fazem necessárias para atender a uma cobertura adequada de procedimentos de reabilitação.

Há necessidade de reorganização dos serviços que fazem dispensação de órteses, próteses e meios auxiliares de locomoção. Diante do quadro apresentado, é evidente que este tipo de atendimento não cobre as diversas regiões do Estado e está em forte discrepância com o que recomenda a portaria no $818 / 2001$.

De forma geral, os gestores dos serviços têm dificuldade em classificar a unidade conforme a portaria, o que traz consequentes problemas na estruturação e na organização da rede de assistência para a reabilitação das pessoas com deficiência e vítimas de acidentes e violências, dificultando a hierarquização e integralidade do atendimento.

\section{Colaboradores}

MLLT Lima, MLC Lima, SF Deslandes, ER Souza e AK Barreira participaram igualmente de todas as etapas de elaboração do artigo. 


\section{Referências}

1. Brasil. Ministério da Saúde (MS). Secretaria de Vigilância em Saúde. Departamento de Análise de Situação em Saúde. Saúde Brasil 2007: uma análise da situação de saúde. Brasília: Ministério da Saúde (MS); 2008.

2. Brasil. Ministério da Saúde (MS). Portaria MS/GM $n^{\circ}$ 737, de 16 de maio de 2001. Dispõe sobre a Política Nacional de Redução da Morbimortalidade por Acidentes e Violências. Diário Oficial da União 2005; 16 mai.

3. Souza ER, Lima MLC. Panorama da violência urbana no Brasil e suas capitais. Cien Saude Colet 2007; 11(Supl.):1211-1222.

4. Gawryszewski VP, Koizumi MS, Mello-Jorge MHP de. As causas externas no Brasil no ano 2000: comparando a mortalidade e a morbidade. Cad Saude Publica 2004; 20(4):995-1003.

5. Organización Pan-Americana de La Salud (OPAS) Violencia y salud: resolución no XIX. Washington: Organización Pan-Americana de La Salud (OPAS) 1994.

6. Gaspar AP, Ingham SJM, Vianna PCP, Santos FPE Chamlian TR, Puertas EB. Avaliação epidemiológica dos pacientes com lesão medular atendidos no Lar Escola São Francisco. Acta Fisiatr 2003; 10(2):73-77.

7. Briceño-León R, organizador. Violencia, sociedad y justicia en América Latina. Buenos Aires: Clacso; 2002.

8. Brasil. Ministério da Saúde (MS). Portaria MS/GM $n^{\circ} 818$, de 5 de junho de 2001. Dispõe sobre a Organização e Implantação de Redes Estaduais de Assistência à Pessoa Portadora de Deficiência Física. Diário Oficial da União 2001; 5 jun.

9. Nallin A. A reabilitação em instituições: suas razões e procedimentos. Análise de representação no dis curso [dissertação]. São Paulo: Universidade de São Paulo; 1992.

10. Organización Pan-Americana de La Salud (OPAS). Desarrollo y fortalecimiento de los sistemas locales de salud en la transformación de los sistemas nacionales de salud. Los Servicios de rehabilitación. Washington: Organización Pan-Americana de La Salud (OPAS); 1993.

11. Organización Pan-Americana de La Salud (OPAS) La reabilitación por discapacidad en América Latina y el Caribe. Bol Sanit Panam 1996; 120(4):358-361.

12. Almeida MC. Saúde e reabilitação de pessoas com deficiência: políticas e modelos assistenciais [tese]. Campinas (SP): Faculdade de Ciências Médicas; 2000

13. Minayo MCS, Deslandes SF, organizadores. Análise diagnóstica da Política Nacional de Saúde para a Redução de Acidentes e Violências. Rio de Janeiro: Fiocruz; 2007.

14. Teixeira CF. Estudo avaliativo da Política de Atenção à Saúde Auditiva: estudo de caso em Pernambuco [tese]. Recife: Centro de Pesquisas Aggeu Magalhães; 2007

15. Furtado BMASM, Araújo Jr JLC, Cavalcanti P. O perfil da emergência do Hospital da Restauração: uma análise dos possíveis impactos após a municipalização dos serviços de saúde. Rev Bras Epidemiol 2004; 7(3):279-289.
16. Cardoso LGRA. Estudo sobre a distribuição dos ser viços de reabilitação: o caso do Estado do Rio de Janeiro [dissertação]. Rio de Janeiro: Escola Nacional de Saúde Pública; 2004.

17. Pernambuco. Plano Estadual de Segurança Pública. [site da Internet]. 2007. [acessado 2009 set 13]. Disponível em: http://www.pactopelavida.pe.gov.br

18. Barata RB, Ribeiro MCSA. Relação entre homicídios e indicadores econômicos em São Paulo, Brasil, 1996. Rev Panam Salud Publ 2000; 7(2):118-124.

19. Bardin L. Análise de conteúdo. Lisboa: Edições 70; 1979.

20. Deslandes SF, Souza ER, Minayo MCS, Costa CRBSF da, Krempel M, Cavalcanti ML, Lima MLC, Moysés SJ, Leal ML, Carmo CN. Diagnostic characterization of services providing care to victims of accidents and violence in five Brazilian state capitals. Cien Saude Colet 2006; 11(2):385-396.

21. Silva SM. Atendimento a pessoa com deficiência é inadequado na maior parte do país. Agência Brasil. Radiobrás [site da Internet]. 2006. [acessado 2007 set 13]. Disponível em: www.agenciabrasil.gov.br/ noticias

22. Brasil. Ministério da Saúde (MS). Secretaria de Atenção à Saúde. Departamento de Ações Programáticas Estratégicas. A pessoa com deficiência e o Sistema Unico de Saúde. Brasília: Ministério da Saúde (MS); 2006.

23. Gavazza CZ, Fonseca VM, Silva KS, Cunha SR Utilização de serviços de reabilitação pelas crianças e adolescentes dependentes de tecnologia de um hospital materno-infantil no Rio de Janeiro, Brasil. Cad Saude Publica 2008; 24(5):1103-1111.

24. Sociedade Brasileira de Medicina Física e Reabilitação. Encontre um especialista. [site da Internet]. [acessado 2009 set 8]. Disponível em: www.fisiatria. org.br

25. Cherem AJ. Medicina física e reabilitação - Fisiatria, um pouco da sua história. Arq Bras Med 1990; 19(2):137-118.

26. Brasil. Ministério da Saúde (MS). Portaria MS/GM $n^{\circ}$ 154, de 24 de janeiro de 2008. Dispõe sobre a Criação dos Núcleos de Apoio à Saúde da Família - Nasf. Diário Oficial da União 2008; 04 mar.

27. França ISX, Pagliuca LMF. Acessibilidade da pessoas com deficiência ao SUS: fragmentos históricos e desafios atuais. Rev Rene Fortaleza 2008; 9(2):129137

Artigo apresentado em 25/10/2009

Artigo aprovado em 21/02/2010

Versão final apresentada em 15/03/2010 